\title{
Landau level splitting in nitrogen-seeded epitaxial graphene
}

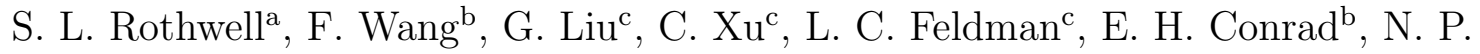 \\ Guisinger $^{\mathrm{d}}$, P. I. Cohen ${ }^{\mathrm{a}}$ \\ ${ }^{a}$ Department of Electrical and Computer Engineering, University of Minnesota, Minneapolis, MN \\ 55455 USA \\ ${ }^{b}$ Department of Physics, Georgia Institute of Technology, Atlanta, GA 30332 USA \\ ${ }^{c}$ Institute for Advanced Materials, Devices, and Nanotechnology, Rutgers University, Piscataway, \\ NJ 08854 USA \\ ${ }^{d}$ Center for Nanoscale Materials, Argonne National Laboratory, Argonne, IL 60439 USA
}

\begin{abstract}
We report scanning tunneling microscopy and spectroscopy (STM and STS) studies of graphene formed from a nitrogen-seeded $\mathrm{SiC}(000 \overline{1})$ surface. STM indicates that much of the graphene consists of wide flat plateaus with hexagonal features bounded by pleats and regions with disordered character. Nitrogen impurities are not observed in the epitaxial graphene layers. STS measurements on this surface show peaks corresponding to Landau levels associated with pseudo-magnetic fields as high as 1000 T. The energy distribution of Landau levels is consistent with an electronic model employing a finite bandgap.
\end{abstract}

\section{Introduction}

Graphene grown epitaxially on silicon carbide presents the intriguing possibility of atomic scale graphene modification by first altering the substrate. Recently we showed that multilayer epitaxial graphene grown on C-face nitrogen-seeded $\mathrm{SiC}$ has a valence band offset of $0.3-0.7 \mathrm{eV}$ [1] from the Fermi level. Here, we examine the morphology and electronic structure of this nitrogenated graphene (NG). Landau levels are clearly 
observed and subsequently interpreted in terms of a pseudo-magnetic field. We show that the sequence of observed levels can be fit with a model that includes a bandgap, consistent with the earlier indications of a gap in this unique material.

Conventional graphene from many fabrication methods, including mechanical exfoliation, SiC epitaxy, and CVD among others, has been characterized extensively reporting high mobilities and no bandgap. Fabricating a semiconducting form of this material may only be achieved by a radical departure from the ideal monolayer sheet. Induced bandgaps in graphene have been reported under a variety of conditions. For example, various dopants [2-6] have been reported to induce a gap, but these films are expected to have low mobility due to scattering from substitutional dopants. An ordered functionalized band gap has been produced in graphene grown on $\mathrm{SiC}(0001)$ but because it is due to an intrinsic surface reconstruction, there is no way to control the gap size [7]. Graphene ribbons have experimentally demonstrated confinement induced bandgaps, but continue to present lithographic challenges to large scale fabrication and device integration $[8,9]$. Graphene placed on a periodic array of topographic features displayed possible small, strain induced gaps of 0.14$0.19 \mathrm{eV}$, dependent on feature pitch [10].

It has been predicted [11-13] and observed $[14,15]$ that a distortion or strain of the graphene lattice will create large pseudo-magnetic fields. However no semiconducting graphene has been reported in association with such strain induced fields. Scanning tunneling spectroscopy (STS) spectra showing several Landau levels induced by pseudo-magnetic fields have been reported for spectra taken across graphene nano bubbles $[14,16]$, graphene on ridges $[15,17]$, planar strained graphene on copper foil 
[18], and molecular graphene assembled from CO [19]. Graphene intercalated with potassium was also reported to have Landau levels which were attributed to varying electrostatic potentials rather than strain [20]. In most of these reports a Landau level at the Dirac point is clearly visible. Scanning tunneling microscopy (STM) and STS results are presented here to determine the Landau level variation over NG films. These results are interpreted in terms of pseudo-magnetic fields inducing a Landau level sequence consistent with a finite bandgap [21, 22].

\section{Nitrogenated Graphene}

As previously reported, nitrogenated graphene was prepared by growth on the carbon-face $\mathrm{SiC}(000 \overline{1})$ on which there were 0.3 monolayers (ML) of $\mathrm{N}$ [1]. These starting surfaces were prepared by oxidation of $\mathrm{SiC}$, followed by annealing in $\mathrm{NO}$, and then etching to leave the submonolayer of $\mathrm{N}$ on the surface [23]. Graphene is then grown on the nitrogen-seeded substrates via controlled silicon sublimation [1, 24, 25]. Between 1 and 8 layers of epitaxial graphene were grown by heating to about 1400 $\mathrm{C}$ in a Si flux [24]. After growth, variable energy X-ray photoelectron spectroscopy (XPS) showed that about 0.2 monolayers (ML) of nitrogen remain at the interface between the $\mathrm{SiC}$ and graphene, strongly bonded to the $\mathrm{SiC}$ substrate. About $25 \%$ of the nitrogen bonds with the graphene and the $\mathrm{SiC}$ in $\mathrm{sp}^{3}$ bonds, indicating there are strong bonds between carbon atoms in the first layer of graphene and carbon atoms in

the substrate. STM indicates that the graphene films are not flat but bend smoothly over nanoscale ripples $[1,25]$.

Angle-resolved photoemission spectroscopy (ARPES) measurements on $50 \mu \mathrm{m}$ 
size regions showed Dirac cones that were broadened and which were shifted from the Fermi level to higher binding energy. These ARPES results at the graphene $K$ point (rotated $30^{\circ}$ from the $\mathrm{SiC}<10 \overline{1} 0>$ direction) show valence band offsets that depend on the number of layers in the epitaxial graphene films. The graphene band structure of a 3 layer film with $0.2 \mathrm{ML}$ interfacial nitrogen content shows an offset from the Fermi level of $0.7 \mathrm{eV}$, while that of an 8 layer film has a smaller $0.45 \mathrm{eV}$ shift. The effective Fermi velocity $\left(v_{F}\right)$ is reduced compared to pristine graphene, consistent with the opening of a bandgap. Both the 3 and 8 layer samples have $v_{F}=0.8 \pm 0.05 \times 10^{6} \mathrm{~m} / \mathrm{s}$.

The ARPES measurements $[1,25]$ from nitrogen-seeded graphene $\pi$ bands are broader in $k$ than for pristine graphene, largely due to the corrugation of the graphene surface shown in Fig. 1. Small modulations in the local graphene height cause a local angular variation in the surface normal. Since the surface normal determines the orientation of the graphene Brillouin zone, the corrugated surface leads to local $k_{x}$ and $k_{y}$ shifts in the $K$-point. This leads to an ARPES image that is an area average of a distribution of parabolic cuts through Dirac cones from locally tilted graphene resulting in an $E$ - and $k$-broadened spectra. Using STS we examine the spatial distribution of the local density of states (LDOS) corresponding to these results but in a way that senses the graphene bending differently.

Ultrafast time resolved terahertz spectroscopy also finds evidence of a bandgap in these NG films. Recently Mihnev et al. [26] showed an increase in the rate of carrier cooling and recombination by over two orders of magnitude. This was attributed to a distribution of finite bandgaps of which a subset was in a range to be bridged by 
optical phonon emission.

It is important to note that the single, linear Dirac cone in the ARPES measurement leads us to conclude that multilayer NG is not electrically coupled between the top layers. If the band structure was from multiple coupled layers, a second cone (or more) would be expected. Bernal stacking would produce a band shifted $0.5 \mathrm{eV}$ from the observed cone to higher binding energy [27], however no other cones shifted in $k_{y}$ are visible. Shifted cones would also be expected because of the rotational stacking of C-face graphene [28]. The lack of these cones means that the rotation angle between the top two layers of graphene captured in the $50 \mu \mathrm{m}$ ARPES beam diameter must be rotated $>10^{\circ}$ with respect to each other (this angle is set by the detector slit window). Such a large relative rotation angle between the two layers is known to keep the layers electronically decoupled $[29,30]$. Thus these multilayer NG films act as electrically uncoupled, multiple, independent graphene layers [31]. Any STS data would be expected to similarly measure the LDOS and electronic features of monolayer graphene.

\section{Results}

STM and STS studies were primarily conducted at the Argonne National Laboratory (ANL) in a UHV variable temperature Omicron STM with an electrochemically etched tungsten tip at room temperature. Reported voltages are sample biases, and constant current mode was used for all images. STS was performed after cooling to 100 K. Images were also taken at Oak Ridge National Laboratory (ORNL) at room temperature. STS and images taken at Brookhaven National Laboratory (BNL) were 
at $4 \mathrm{~K}$. As seen in Fig. 1, STM on NG films reveals a topography of pleats and
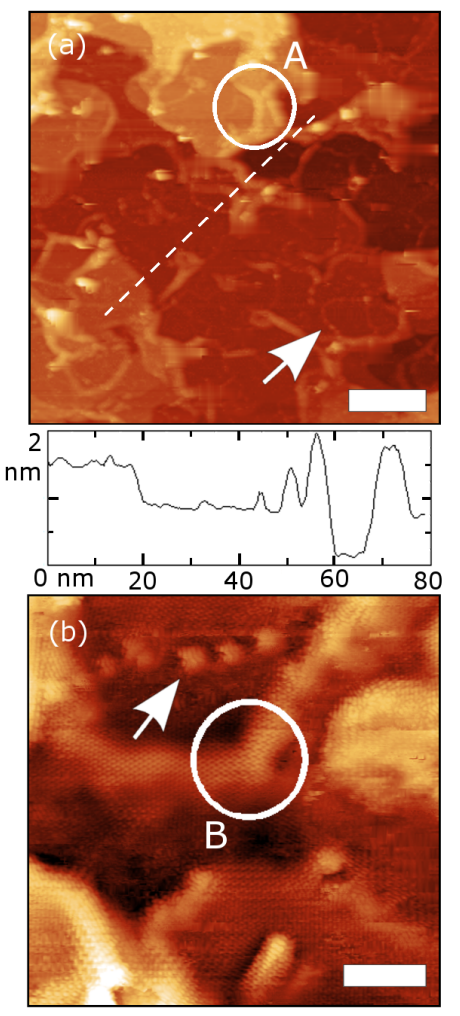

Figure 1: Scanning tunneling microscopy of graphene grown on C-face nitrogenated SiC. (a) Overview of 4 layer NG showing plateaus and smooth pleats. Circle A highlights pleats at the edge of a plateau. $20 \mathrm{~nm}$ scale bar, $1 \mathrm{~V}, 0.03 \mathrm{nA}$. A topographic profile following the dotted line is plotted below. (b) Atomic resolution of a region with ripples and nanocaps from $0-1 \mathrm{~nm}$ in height. Circle B highlights a single ripple. 4 layer NG, $3 \mathrm{~nm}$ scale bar, $0.1 \mathrm{~V}, 1 \mathrm{nA}$. Images taken at ORNL (a) and ANL (b).

plateaus. Several topographic regimes are visible: Plateaus appear as atomically flat islands with partial hexagonal features characteristic of graphene. Even on thicker samples the plateaus are flat enough that moiré patterns are sometimes visible, a common feature on multilayer $\mathrm{C}$-face $\mathrm{SiC}$ graphene $[24,32]$. Plateaus are typically bounded by smooth pleats at the edges which may roll over onto a subsequent plateau. Circle A in Fig. 1(a) highlights smooth pleats at a plateau edge. Pleats are typically up to $\sim 2 \mathrm{~nm}$ high, $\sim 1-4 \mathrm{~nm}$ wide, and 2-20 nm long. Rippled regions are sometimes seen separating plateaus or within a plateau, particularly on thicker samples. 
Fig. 1(b) displays atomic resolution over an entire rippled area (lower left), and circle B highlights a specific ripple with atomic resolution. Despite the irregular surface, atomic resolution such as in Fig. 1(b) can often be achieved on pleats and ripples as well as flat plateaus showing a defect free lattice flowing smoothly from one plateau to another. Finally some areas have distinctly rougher $\sim 1-5 \mathrm{~nm}$ features which are difficult to image due to lower conductivity and low material stability. This spherical debris may be seen in Fig. 1(a) along the top edge as a rougher texture on the top plateau. Nanocaps are also visible, indicated in Fig. 1(a) and (b) by arrows. Nanocaps are not seen on pristine controlled silicon sublimation (CSS) C-face graphene without nitrogen, but have been seen at a lower density in graphene grown on C-face $\mathrm{SiC}$ in Argon or UHV [32-34]. Larger pleats within larger flat regions are seen on pristine CSS C-face graphene, again at a much lower density [24].

Substitutional nitrogen atoms in the graphene lattice are expected to have characteristic scattering patterns visible in STM images. However, in support of previously reported XPS analyses placing the nitrogen below the graphene, no scattering patterns indicating substitutional nitrogen were seen in NG [35-37]. Since XPS showed the interfacial nitrogen to be in $\mathrm{sp}^{3}$ bonds, the nitrogen apparently pins the graphene to the substrate during growth, causing the film to buckle and form the observed wrinkles [1]. The line profile in Fig. 1 captures typical pleat and step heights along the dashed line in the top panel. The step heights in this profile, as well as others not shown, indicate that often changes in NG surface height are created by $\mathrm{SiC}$ steps combined with graphene steps. This implies that changes in the surface topography are not solely the result of changes in graphene thickness. Furthermore, the graphene 
is able to conform to and maintain the substrate step profile or rippled morphology even after 4 layers as in Fig. 1. Note that the interfacial nitrogen remains at the interface as $\mathrm{SiC}$ is consumed in the graphene growth process.

Each STS spectrum is found by taking a derivative of current with respect to voltage as the STM tip is held fixed at one location and height, and is proportional to the LDOS in that area [38]. Fig. 2(a) shows four typical NG STS spectra taken within $2 \mathrm{~nm}$ of the pleat at the edge of a plateau. Fig. 3 shows the observed spatial variation in NG STS spectra across a $0.5 \mathrm{~nm}$ high ripple. Both figures display spectra vertically shifted for clarity. Following Levy et al. and others $[14,15,17]$ we identify the symmetric peaks as Landau levels due to a pseudo-magnetic field. The peaks are indexed with integer values of $n$ and are fit to energies proportional to $\sqrt{n}$ since the ARPES measurements show independent, single layer graphene electronic behavior. Importantly, our spectra consistently lack a central peak near $0 \mathrm{~V}$ close to the expected Dirac point. In contrast, other experimental measurements of pseudo-magnetic field induced Landau levels in graphene typically show a single, clearly visible central peak at the Dirac point [14-17].

A bandgap in graphene may be modeled in the Dirac Hamiltonian by adding diagonal terms, $\pm \frac{\Delta}{2}$. The positions of Landau levels in a magnetic field are then given by:

$$
E-E_{D}= \pm \sqrt{2 e \hbar v_{F}^{2} B|n|+\frac{\Delta^{2}}{4}},
$$

where $e$ is the electron charge, $v_{F}$ the Fermi velocity, $B$ the pseudo-magnetic field magnitude, $n$ the integer Landau index, and \pm for the valence and conduction bands 

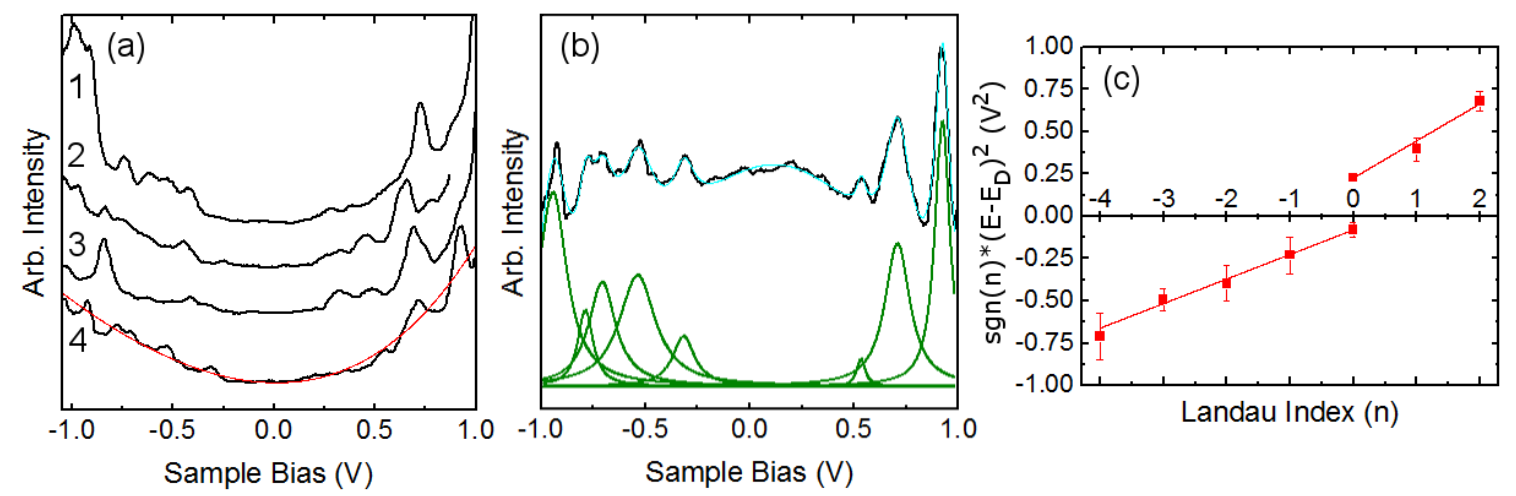

Figure 2: Scanning tunneling spectroscopy of NG graphene, spectra vertically offset for clarity. (a) STS spectra (black) at different points near the edge of a plateau; the spectrum 4 is fit with a polynomial background (red). (b) Residual after background subtraction (black) and Lorentzian peak fitting (cyan: total fit, green: individual Lorentzian peak fits). (c) Peak energy squared plotted against $n$, assuming a bandgap. The measured pseudo-magnetic field magnitude is $256 \pm 12 \mathrm{~T}$ and $278 \pm 27 \mathrm{~T}$ for the valence and conduction bands respectively. STS taken at Brookhaven National $\mathrm{Lab}(\mathrm{BNL})$ at $4 \mathrm{~K}$.

respectively $[12,39,40]$. Each of these levels is expected to have the same number of states [41]. By this model, a gap splits the ground state Landau level into two $n=0$ peaks for valence and conduction band such that $E_{g a p}=\Delta$. No peak is visible at the Dirac point, $E_{D}$, which is assumed to be in the center of the gap.

Fig. 2 illustrates the method used to extract the pseudo-magnetic field magnitudes in NG [14]. The STS spectra in Fig. 2(a) were measured on a flat region, near the edge of a plateau. The background is fit with a fourth order polynomial (red) following Levy et al. [14] since the envelope and background do not correspond to the expected sum of broadened Lorentzians [42]. It provides a well-defined procedure for identifying the peak positions. The top curve in Fig. 2(b) shows the residual spectrum for spectrum 4 after subtraction of the polynomial background. The bottom curves show Lorentzian fits to the peaks (green). The peaks are symmetric with widths of the order of $50-250 \mathrm{meV}$. Note that for our data this procedure was not necessary for determination of the peak positions but allowed us to determine the shape of the 

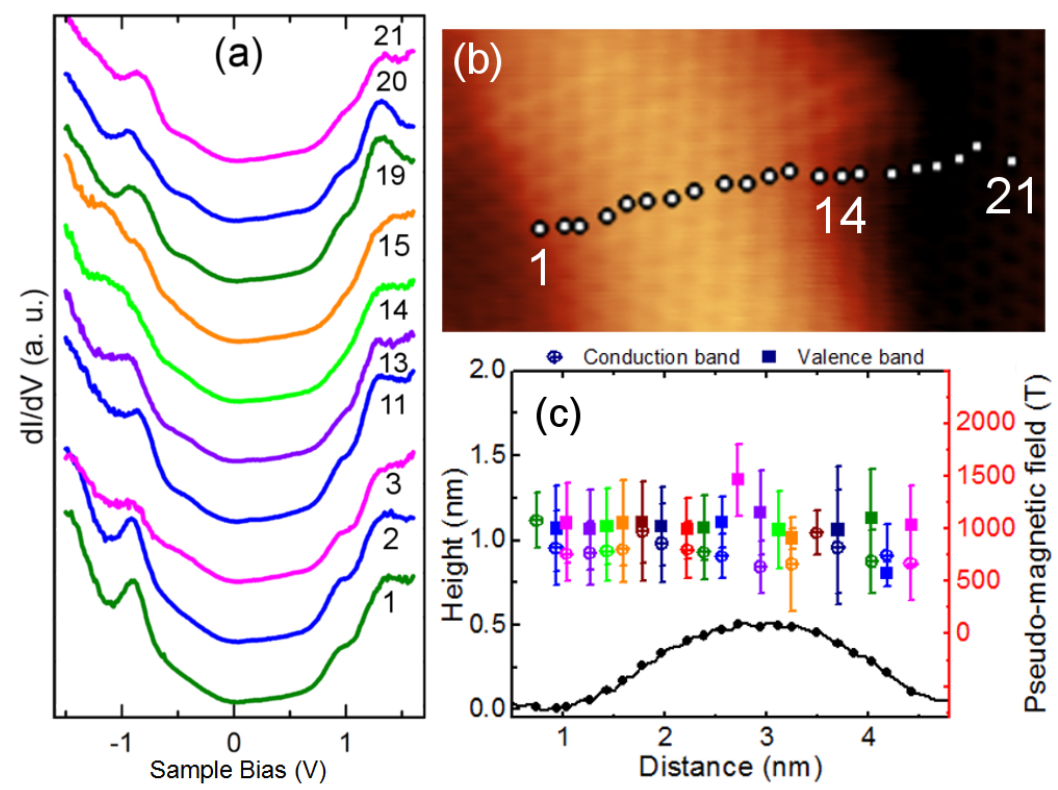

Figure 3: STS taken across a $0.5 \mathrm{~nm}$ high ripple. (a) Progression of STS spectra corresponding to pseudo-magnetic field values in (c) and positions in (b). (b) STM topography image with STS locations marked. (c) Pseudo-magnetic field magnitudes plotted against ripple profile. STS taken at ANL at $100 \mathrm{~K}$.

peaks. Fig. 2(c) plots the square of the peak energies ( \pm for valence or conduction band) of the bottom spectrum against Landau index $n$; the expected linearity is evident. For the plots, the Dirac point is chosen to be at the midpoint between the two $n=0$ levels. The Fermi velocity, $v_{F}=0.8 \times 10^{6} \mathrm{~m} / \mathrm{s}$ measured via ARPES [1], is used to determine the pseudo-magnetic field magnitude for both bands of spectrum 4 in Fig. 2 giving $256 \pm 12 \mathrm{~T}$ for the valence band and $278 \pm 27 \mathrm{~T}$ for the conduction band. The pseudo-magnetic field magnitude varies between 160-300 $\mathrm{T}$ for all four spectra in Fig. 2. This analysis is further expanded upon in the discussion section.

Fig. 3 shows a series of STS spectra taken across a typical $0.5 \mathrm{~nm}$ high ripple, found by zooming into a rippled region (similar to Fig. 1(b)). Each spectrum was taken multiple times to confirm reproducibility, and was analyzed as described in Fig. 2 with the removal of a polynomial background and Lorentzian peak fitting. The 
spectra are vertically shifted for clarity and have numbers corresponding to positions indicated on the STM image in Fig. 3(b) and (c). The measured pseudo-magnetic field magnitudes are plotted in Fig. 3(c) vs distance across the ripple along with the height of the ripple as measured by STM. The error bars are from variance in the linear slope of index vs energy squared, originating from the width of the Lorentzian peaks used to fit each Landau level.

Electrostatic potential variations over the graphene would be expected to measurably shift the Dirac point in our STS spectra. Although the point midway between conduction and valence band peaks does shift by $\pm 0.5 \mathrm{~V}$ in our measurements, we could not find a correlation between these values and topography, pseudo-magnetic field magnitude, or features in STM images.

\section{Discussion}

None of the STS spectra displayed in Figs. 2 or 3 show the topologically protected peak at the Dirac point. There are two models which seem theoretically applicable for this: (1) The STS spectra may have a central $n=0$ peak of such low intensity that the peak is not visible above the noise threshold, or (2) the spectra may have a split $n=0$ peak which is posited to occur in graphene with a bandgap induced by two perturbations $[21,22]$. For the first analysis model, the two peaks closest to $0 \mathrm{~V}$ in an NG spectrum would be analyzed as the $n= \pm 1$ peaks. For the second, the first peaks would be considered the respective $n=0$ peaks for the valence and conduction band. Fig. 4 illustrates these alternate models using the four spectra from Fig. 2 by plotting peak energy squared against the Landau index for the two cases. As may 

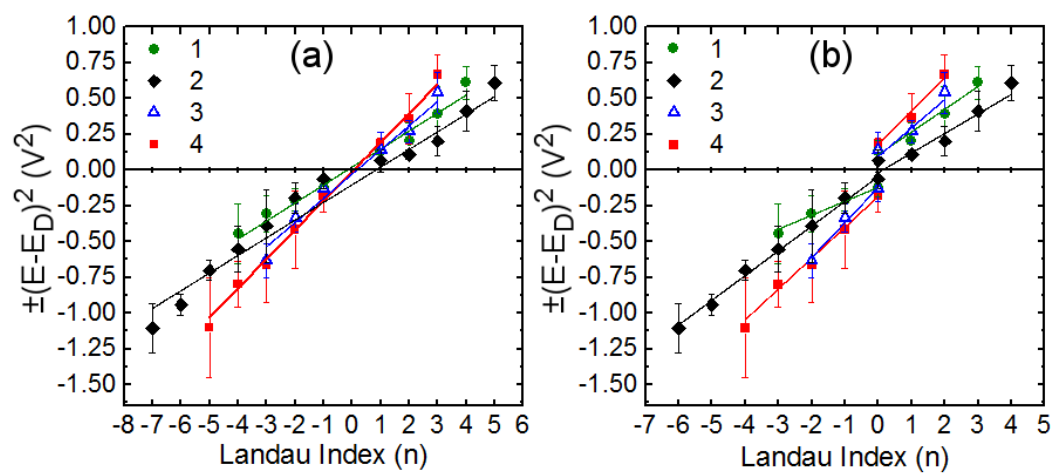

Figure 4: Comparison between two different analyses of peak energy squared vs. Landau index for the four spectra from Fig. 2. (a) Peaks are indexed with a missing $n=0$ level. (b) Peaks are indexed with a split $n=0$ level, including a bandgap in the model.

be seen, both analysis procedures give excellent linearity and from this comparison alone it is difficult to say which model fits the data better. The pseudo-magnetic field magnitudes determined in Fig. 4 are similar. Using either model, the pseudo-magnetic field magnitude ranges from 100-300 T.

Theory suggests that Landau levels should have the same number of states regardless of index [41], which would imply that apart from variation introduced by broadening [42] a very similar intensity is expected for all Landau peaks. Experimental data on Landau levels induced by applied magnetic fields in graphene do show $n=0$ central peaks with equivalent intensity to higher index peaks [42]. Further, Landau levels in strained graphene $[14,15]$ and in graphene-like graphite $[17,20]$ have been reported before but in those cases the $n=0$ peak is the most intense or comparable to the higher energy peaks, which is also different than the trend seen here. These cast doubt on the likelihood of the first analysis model examined in Fig. 4.

For Landau levels, the ground state $n=0$ peak is doubly degenerate and requires two perturbations to break the symmetry (thereby inducing a bandgap). Pseudo- 
magnetic fields alone are insufficient to break the double degeneracy; another perturbation, such as an electrostatic field, must be present [21, 22, 39]. While the existence of strain giving rise to a pseudo-magnetic field is clearly consistent with the data, the origin of a second perturbation, such as an electrostatic field, is not yet known. Nevertheless, a split $n=0$ peak is consistent with our previous ARPES measurement indicating an energy gap below the Fermi level. For these reasons the second analysis procedure seems most logical; both Fig. 2 and 3 use this procedure to find the reported pseudo-magnetic field magnitudes.

Having concluded that analyzing the STS spectra with a bandgap is most consistent, let us now consider the theoretical predictions of pseudo-magnetic field variation with topographically induced strain. The strain tensor for a topographic height profile $h(y)$ may be estimated from:

$$
u_{y y}=\frac{\partial u_{y}}{\partial y}+\frac{1}{2}\left(\frac{\partial h}{\partial y}\right)^{2} \approx \frac{1}{2}\left(\frac{\partial h}{\partial y}\right)^{2}
$$

Using this approximation, a theoretical estimate of the pseudo-magnetic field follows from $B \propto-\partial u_{y y} / \partial y[15,43,44]$. If we consider the profile in Fig. 3 as a Gaussian 1D pleat, we obtain a maximum field of about $800 \mathrm{~T}$, the same order of magnitude as the measured field of about $1000 \mathrm{~T}$.

Since the strain in NG films develops during growth, we expect both positive and negative strain gradients, and hence positive and negative pseudo-magnetic fields which vary in magnitude and cross zero in between. Although STS spectra only measure an absolute value of the pseudo-magnetic field magnitude, we expect to see a decrease toward zero field and subsequent increase. Averaging over variation in 
local pseudo-magnetic fields could obscure this expected trend. For the $1000 \mathrm{~T}$ field measured in Fig. 3, the extent of the Landau level's wavefunction is of order $2 \sqrt{2 \hbar / e B}$ [45], or $\sim 2.3 \mathrm{~nm}$. Since the ripple featured in Fig. 3 is only $4 \mathrm{~nm}$ wide, only small changes in the measured field would be expected. Indeed, we measured the pseudomagnetic field magnitude to vary only $\pm 500 \mathrm{~T}$ across the feature. Fig. 2 displays spectra from locations on the flat area of a plateau and also on an edge pleat. It seems reasonable to consider the pleats to be under compressive strain, and the flat plateau area under tensile strain, if any. Despite the expected difference in strain, the variation in pseudo-field magnitude for Fig. 2 is no more than $\pm 90 \mathrm{~T}$ around an average value of $200 \mathrm{~T}$. Similar instances where variation in pseudo-magnetic field magnitude is expected but not experimentally observed have been reported by others $[14,15]$.

If the STS measurement averages over overlapping Landau wavefunctions, then it is reasonable to expect a contribution to the Landau level peak widths from the range of pseudo-magnetic field magnitudes in the surrounding region. As a rough estimate, the observed broadening from Eq. 1 should be of the order $\delta E=e \hbar v_{F}^{2}|n| \delta B /\left(E-E_{D}\right)$. The peak widths for the bottom spectrum of Fig. 2(b) vary and range from 50$250 \mathrm{meV}$ for a measured field of $\sim 160-300$ T. In Fig. 3 there is similar amount of broadening for a measured field of $\sim 1000 \mathrm{~T}$. Some of this broadening appears to be an artifact of the peak fitting procedure used to determine the energy locations. Nevertheless, for the level of peak broadening observed in Figs. 2 and 3 even with the uncertainties, the pseudo-magnetic field variation, $\delta B$, should be no more than about $30 \mathrm{~T}$. This would seem to imply that a change in pseudo-magnetic field polarity 
would be visible via STS, even with averaging smoothing the data. Nevertheless, no zero crossing or minimum was observed in either Fig. 2 or 3.

These results may be related to the STS measurements reported by Guo et al. [20] in which Landau levels were observed in potassium intercalated graphite. In this system, Landau levels were also observed without an applied field though their peak heights had a very different overall envelope. Further, their Dirac point was found to be far from the Fermi level. They suggested that the measured pseudomagnetic field could be due to a strain related vector potential. In a couple of cases shown in their supplementary data, STS spectra with a small number of Landau levels exhibited a bandgap similar to that observed in Fig. 2. However, if the Dirac point was positioned as their other data, then the mechanism is different from that reported here. For our data, which always exhibited a gap, the Dirac point was found by fitting to be midway between the two $n=0$ peaks, in agreement with our ARPES data on similarly prepared films.

\section{Conclusion}

In conclusion, previous work indicated that $\mathrm{NG}$ has a valence band shift of $>0.7 \mathrm{eV}$, a high carrier recombination rate, a topography consisting of many pleats, and a small nitrogen layer at the $\mathrm{SiC} /$ graphene interface. Strain induced by these nitrogen bonds was suspected to play a role in the formation of the bandgap. To examine this mechanism and to further characterize these NG films we have used STM and STS to

measure their structure and electronic properties. We have observed Landau level type peaks via STS essentially everywhere on the surface of NG films. No peaks were 
observed in STS spectra near the Dirac point, consistent with a bandgap and the splitting of the $n=0$ level by two perturbations. The expected spatial variation in the measured pseudo-magnetic field was not observed. A strong $\sqrt{n}$ dependence was observed in peak energy when a gap of $\sim 1 \mathrm{eV}$ was included. No sharp midgap states that could give rise to recombination were observed. In short, our STM and STS measurements of NG films are consistent with previous ARPES and THZ recombination measurements finding a bandgap in nitrogenated graphene.

\section{Acknowledgments}

We are grateful to T. Low for insightful discussions, and P. Zahl, and J. Wang for assistance in the data acquisition and analysis. We acknowledge funding from NSF-DMR 1206793, 1206655, 1606256. Use of the Center for Nanoscale Materials at Argonne was supported by the U. S. Department of Energy, Office of Science, Office of Basic Energy Sciences, under Contract No. DE-AC02-06CH11357. This research was also carried out in part at the Center for Functional Nanomaterials, Brookhaven National Laboratory, which is supported by the U.S. Department of Energy, Office of Basic Energy Sciences, under Contract No. DE- SC0012704. Another portion of this research was conducted at the Center for Nanophase Materials Sciences, which is a DOE Office of Science User Facility at Oak Ridge National Laboratory.

\section{References}

[1] F. Wang, G. Liu, S. Rothwell, M. Nevius, A. Tejeda, A. Taleb-Ibrahimi, L. Feldman, P. Cohen, E. Conrad, Wide-gap semiconducting graphene from nitrogenseeded SiC, Nano Lett. 13 (2013) 4827-4832. 
[2] J. Robinson, J. Burgess, C. Junkermeier, S. Badescu, T. Reinecke, F. Perkins, M. Zalalutdniov, J. Baldwin, J. Culbertson, P. Sheehan, E. Snow, Properties of fluorinated graphene films, Nano Lett. 10 (2010) 3001-3005.

[3] M. Deifallah, P. McMillan, F. Corà, Electronic and structural properties of twodimensional carbon nitride graphenes, J. Phys. Chem. C 112 (2008) 5447-5453.

[4] A. Lherbier, X. Blase, Y.-M. Niquet, F. Triozon, S. Roche, Charge transport in chemically doped 2D graphene, Phys. Rev. Lett. 101 (2008) 036808.

[5] H. Liu, Y. Liu, D. Zhu, Chemical doping of graphene, J. Mater. Chem. 21 (2011) $3335-3345$.

[6] C. Coletti, C. Riedl, D. S. Lee, B. Krauss, L. Patthey, K. von Klitzing, J. H. Smet, U. Starke, Electronic structure of nitrogen-carbon alloys a-CNx determined by photoelectron spectroscopy., Phys. Rev. B 81 (2010) 235401.

[7] M. S. Nevius, M. Conrad, F. Wang, A. Celis, M. N. Nair, A. Taleb-Ibrahimi, A. Tejeda, E. Conrad, Semiconducting graphene from highly ordered substrate interactions, Phys Rev Lett. 115 (2015) 136802.

[8] Z. Chen, Y. Lin, M. Rooks, P. Avouris, Graphene nano-ribbon electronics, Physica E 40 (2007) 228-232.

[9] M. Y. Han, B. Ozyilmaz, Y. Zhang, P. Kim, Energy band-gap engineering of graphene nanoribbons, Phys. Rev. Lett. 98 (2007) 206805.

[10] J.-K. Lee, S. Yamazaki, H. Yun, J. Park, G. P. Kennedy, G.-T. Kim, O. Pietzsch, 
R. Wiesendanger, S. Lee, S. Hong, U. Dettla-Weglikowska, S. Roth, Modulation of electronic structure of graphene by substrate induced nanomodulation, Nano Lett. 13 (2013) 3494-3500.

[11] F. Guinea, T. Low, Band structure and gaps of triangular graphene superlattices, Phil. Trans. R. Soc. A 368 (2010) 5391-5402.

[12] M. Vozmediano, M. Katsnelson, F. Guinea, Gauge fields in graphene, Phys. Rep. 496 (2010) 109-148.

[13] D. Moldovan, M. Masir, F. Peeters, Electronic states in a graphene flake strained by a Gaussian bump, Phys. Rev. B 88 (2013) 035446.

[14] N. Levy, S. A. Burke, K. L. Meaker, M. Panlasigui, A. Zettl, F. Guinea, A. H. Castro Neto, M. F. Crommie, Strain-induced pseudo-magnetic fields greater than 300 Tesla in graphene nanobubbles, Science 329 (2010) 544-547.

[15] L. Meng, W. Y. He, H. Zheng, M. Liu, H. Yan, W. Yan, Z. D. Chu, K. Bai, R. F. Dou, Y. Zhang, Z. Liu, J. C. Nie, L. He, Strain-induced one-dimensional Landau level quantization in corrugated graphene, Phys. Rev. B 87 (2013) 205405.

[16] J. Lu, A. H. Castro Neto, K. P. Loh, Transforming moiré blisters into geometric graphene nano-bubbles, Nat. Commun. 3 (2012) 823 (6pp).

[17] H. Yan, Y. Sun, L. He, J.-C. Nie, M. H. W. Chan, Observation of Landau-levellike quantization at $77 \mathrm{~K}$ along a strained-induced graphene ridge, Phys. Rev. B 85 (2012) 035422. 
[18] N.-C. Yeh, M.-L. Teague, S. Yeom, B. Standley, R.-P. Wu, D. Boyd, M. Bockrath, Strain induced pseudo-magnetic fields and charging effects on CVD-grown graphene, Surf. Sci. 605 (2011) 1649-1656.

[19] K. K. Gomes, W. Mar, W. Ko, F. Guinea, H. C. Manoharan, Designer Dirac fermions and topological phases in molecular graphene, Nature 483 (2012) 306310.

[20] D. Guo, T. Kondo, T. Machida, K. Iwatake, S. Okada, J. Nakamura, Observation of Landau levels in potassium-intercalated graphite under a zero magnetic field, Nat. Commun. 3 (1068) (2012) 1-6.

[21] T. Low, F. Guinea, M. I. Katsnelson, Gaps tunable by electrostatic gates in strained graphene, Phys. Rev. B 83 (2011) 195436.

[22] I. Snyman, Gapped state of a carbon monolayer in periodic magnetic and electric fields, Phys. Rev. B 80 (2009) 054303.

[23] S. Dhar, Nitrogen and hydrogen induced trap passivation at the $\mathrm{SiO}_{2} / 4 \mathrm{H}-\mathrm{SiC}$ interface, Ph.D. thesis, Vanderbilt University, 2005.

[24] W. A. de Heer, C. Berger, M. Ruan, M. Sprinkle, X. Li, Y. Hu, B. Zhang, J. Hackinson, E. H. Conrad, Large area and structured epitaxial graphene produced by confinement controlled sublimation of silicon carbide, Proc. Natl. Acad. Sci. 108 (41) (2011) 16900-16905.

[25] F. Wang, G. Liu, S. L. Rothwell, M. S. Nevius, C. Mathieu, N. Barrett, A. Sala, T. Menteş, A. Locatelli, P. I. Cohen, L. C. Feldman, E. H. Conrad, Pattern in- 
duced ordering of semiconducting graphene ribbons grown from nitrogen-seeded SiC, Carbon 82 (2015) 360-367.

[26] M. T. Mihnev, F. Wang, G. Liu, S. Rothwell, P. I. Cohen, L. C. Feldman, E. H. Conrad, T. B. Norris, Evidence for bandgap opening in buckled epitaxial graphene from ultrafast time-resolved terahertz spectroscopy, Appl. Phys. Lett. 107 (17) 173107, doi:10.1063/1.4934781.

[27] T. Ohta, A. Bostwick, J. L. Mcchesney, T. Seyller, K. Horn, E. Rotenberg, Interlayer interaction and electronic screening in multilayer graphene investigated with angle-resolved photoemission spectroscopy, Phys. Rev. Lett. 98 (20) (2007) 206802, ISSN 0031-9007, doi:10.1103/PhysRevLett.98.206802.

[28] J. Hass, F. Varchon, J. E. Millan-Otoya, M. Sprinkle, N. Sharma, W. A. De Heer, C. Berger, P. N. First, L. Magaud, E. H. Conrad, Why multilayer graphene on 4H-SiC (0001) behaves like a single sheet of graphene, Phys. Rev. Lett. 100 (12) (2008) 125504, ISSN 0031-9007, doi:10.1103/PhysRevLett.100.125504.

[29] J. M. B. Lopes dos Santos, N. M. R. Peres, A. H. Castro Neto, Graphene bilayer with a twist: electronic structure., Phys. Rev. Lett. 99 (2007) 256802, doi:10.1103/PhysRevLett.99.256802.

[30] W. A. de Heer, C. Berger, X. Wu, M. Sprinkle, Y. Hu, M. Ruan, J. A. Stroscio, P. N. First, R. Haddon, B. Piot, C. Faugeras, M. Potemski, J.-S. Moon, Epitaxial graphene electronic structure and transport, J. Phys. D: Appl. Phys. 43 (2010) 374007 (13pp). 
[31] M. Sprinkle, D. Siegel, Y. Hu, J. Hicks, A. Tejeda, A. Taleb-Ibrahimi, P. L. Fèvre, F. Bertran, S. Vizzini, H. Enriquez, S. Chiang, P. Soukiassian, C. Berger, W. de Heer, A. Lanzara, E. Conrad, First direct observation of nearly ideal graphene band structure, Phys. Rev. Lett. 103 (2009) 226803.

[32] J. R. Hass, Structural characterization of epitaxial graphene on SiC, Ph.D. thesis, Georgia Institute of Technology, 2008.

[33] M. Naitoh, M. Kitada, S. Nishigaki, N. Toyama, F. Shoji, An STM observation of the initial process of graphitization at the $6 \mathrm{H}-\mathrm{SiC}(000 \overline{1})$ surface, Surf. Rev. Lett. 10 (2 \& 3) (2003) 473-477.

[34] C. Riedl, Epitaxial graphene on silicon carbide surfaces: growth, chracterization, doping and hydrogen intercalation, Ph.D. thesis, Friedrich-Alexander-Universität Erlangen-Nürnburg, 2010.

[35] F. Joucken, Y. Tison, J. Lagoute, J. Dumont, D. Cabosart, B. Zheng, V. Repain, C. Chacon, Y. Girard, A. R. Botello-Méndez, S. Rousset, R. Sporken, J.-C. Charlier, L. Henrard, Localized state and charge transfer in nitrogen-doped graphene, Phys. Rev. B 85 (2012) 161408.

[36] N. P. Guisinger, G. M. Rutter, J. N. Crain, C. Heiliger, P. N. First, J. A. Stroscio, Atomic scale investigation of graphene formation on $6 \mathrm{H}-\mathrm{SiC}$ (0001), J. Vac. Sci. and Technol. A 26 (932) (2008) 932-937.

[37] R. Lv, Q. Li, A. Botello-Méndez, T. Hayashi, B. Wang, A. Berkdemir, Q. Hao, A. L. Elías, R. Cruz-Silva, H. R. Gutiérrez, Y. A. Kim, H. Muramatsu, J. Zhu, 
M. Endo, H. Terrones, J.-C. Charlier, M. Pan, M. Terrones, Nitrogen-doped graphene: beyond single substitution and enhanced molecular sensing, Sci. Reports 2 (2012) $586(8 \mathrm{pp})$.

[38] C. J. Chen, Theory of scanning tunneling spectroscopy, J. Vac. Sci. Technol. A 6 (2) (1988) 319-322.

[39] X. Li, F. Zhang, Q. Niu, Unconventional quantum hall effect and tunable spin hall effect in Dirac materials: application to an isolated $\mathrm{MoS}_{2}$ trilayer, Phys. Rev. Lett. 110 (2013) 066803.

[40] M. Koshino, T. Ando, Physics of Graphene, Springer, 188, 2014.

[41] M. I. Katsnelson, Graphene: carbon in two dimensions, Cambridge University Press, iSBN 978-0-521-195-19540-9, 2012.

[42] E. Y. Andrei, G. Li, X. Du, Electronic Properties Of Graphene: A Perspective From Scanning Tunneling microscopy and magnetotransport, Rep. Prog. Phys. 75 (5) (2012) 056501 (47pp), ISSN 0034-4885, doi:10.1088/0034$4885 / 75 / 5 / 056501$.

[43] M. Neek-Amal, F. M. Peeters, Strain-engineered graphene through a nanostructured substrate. II. Pseudomagnetic fields, Phys. Rev. B 85 (19) (2012) 195446.

[44] Y. Jiang, T. Low, K. Chang, M. I. Katsnelson, F. Guinea, Generation of pure bulk valley current in graphene, Phys. Rev. Lett. 110 (4) (2013) 046601. 
[45] E. McCann, Graphene Nanoelectronics, chap. 8: Electronic properties of monolayer and bilayer graphene, Springer, 259, 2012. 